\title{
Original
}

\section{Experimental Verification of Finite Element Analysis for a Thermoplastic Orthodontic Aligner}

\author{
Yuko Fujita $^{* 1)}$, Hitoshi KimurA ${ }^{2)}$, Wakana YanAGISAWA ${ }^{1)}$, \\ Norio INOU $^{2)}$ and Koutaro MAKI ${ }^{1)}$
}

\begin{abstract}
In recent years, good outcomes have been reported using transparent and removable orthodontic appliances known as aligners. However, unpredicted tooth movement that contradicted 3-dimensional image simulations was observed in some cases. These anomalies could relate to biomechanical factors; in particular, the characteristics of mechanical loading applied to the periodontal ligament and the tooth crown by aligners remain unclear. This study examines the biomechanical characteristics of aligners by a new method as follows : 1) development of an experimental model using artificial teeth and plastic aligners;2) finite element (FE) modeling and analysis using computed tomography (CT) images of the experimental model; and, 3) comparison among observations of this actual model and standard FE analysis results. Roots of two artificial teeth were covered by silicone material at $1.0 \mathrm{~mm}$ intervals for each coronal proximal surface and plastic clear aligners were manufactured based on another model in which the interval was reduced to $0.0 \mathrm{~mm}$ to simulate bodily movement. An FE analysis model of this $1.0 \mathrm{~mm}$ teeth interval was reconstructed from the CT images. A virtual aligner based on the FE model was also generated with a $0.0 \mathrm{~mm}$ interval. Changes in space between the root surface and silicone in both the actual and FE model were compared with the aligner fitted in the initial model. Identical tendencies of movement were observed in both experimental results - the artificial teeth and computational results of FE analysis. Our method using an experimental and computational approach proved useful to examine aligner characteristics; the use of such a biomechanical approach could further our understanding of aligner treatments.
\end{abstract}

Key words : orthodontics, aligner, finite element, biomechanics, thermoplastic

\section{Introduction}

The field of orthodontics has been evolving ever since the mid-1800s. In the 1890s, Dr. Edward H. Angle formally organized orthodontics as a dental specialty ${ }^{1)}$. This historical event paved the way for orthodontic treatment using braces, orthodontic wires, and brackets, which have been used for some time. Several studies have analyzed the wire treatments mechanically

\footnotetext{
1) Department of Orthodontics, Showa University School of Dentistry, 2-1-1 Kitasenzoku, Outa-ku, Tokyo 145-8515, Japan.

2) Department of Mechanical Engineering and Science, Tokyo Institute of Technology.

* To whom corresponding should be addressed.
} 
by several different techniques, including the photoelastic method ${ }^{2)}$, the strain gauge $\operatorname{method}^{3)}$, and the finite element (FE) analysis method ${ }^{4-9)}$. Studies using FE analysis have elucidated the points of force on teeth, force strength, and direction.

In recent years, esthetic orthodontic treatments using aligners have gained popularity. One such aligner that is frequently chosen is the Invisalign ${ }^{@}$ (hereinafter referred to as "aligner/ s”) aligner developed by Align Technology, Inc. (San Jose, California, US) in 1999 ${ }^{10)}$. This device makes a three-dimensional (3D) simulation from patients' dental casts and simulates the process required to move each tooth into the correct position. However, in some cases, tooth movement does not follow the pre-treatment movement simulation $\left(\right.$ Clincheck $\left.^{\circledR}\right)$, possibly because the mechanism of tooth movement caused by aligners characteristically differs from that of conventional treatment using wires. Aligners cover the entire tooth crown, making it difficult to determine the sites of force and moment application.

A previous study investigated the moment mechanism ${ }^{11)}$, although only the tooth crown area was analyzed. Therefore, the state of stress on the tooth root area remains unclear. More accurate treatment simulations require clarification of the state of mechanical loading placed on the tooth root by aligners. In this study, we attempted to build an FE model that more accurately represents the orthodontic force placed on teeth by aligners. We subsequently validated this model by comparison with an experimental model using actual artificial teeth.

\section{Methods}

Aligners and teeth are completely separate items, thus it will always be difficult to analyze their interaction using an FE model. Therefore, we designed a separate model for the aligners so that they could cover teeth similar to those encountered in standard clinical practice. Both models had a contact relationship. This contact analysis was an analysis of how two adjacent models move separately without any common joints.

To confirm the validity of this FE analysis, an artificial tooth alignment model was created and the results of the artificial tooth alignment model were compared with those of the FE analysis.

(1) Two artificial teeth (canine and second molar) were fixed at 2.5 times the size of actual teeth (B10-330, Nissin Dental Products, Kyoto, Japan) (Fig. 1a), and then fixed into standardized positioning trays using resin.

(2) The distances between the two tooth crowns were set at $0.0 \mathrm{~mm}$ (Fig. 1b) and $1.0 \mathrm{~mm}$.

(3) Tooth crowns were shaped by casting with dental impression silicone (FUSION II flow type, GC CORPORATION, Tokyo, Japan) (Fig. 1c, Fig. 1d).

(4) A plaster cast and artificial tooth model were subsequently fabricated based on the casted silicone. First, the plaster casts were created by pouring dental plaster into the silicone crown castings with coronal proximal surface intervals of $0.0 \mathrm{~mm}$ (Fig. 2a).

(5) The plaster casts were removed, and a plastic sheet (DURAN ${ }^{@}$, SCHEU Dental Technology, Iserlohn, Germany) was used for suction pressing of the crown section, thereby recreating an aligner (Fig. 2b).

(6) The artificial tooth model involved fitting the $2.5 \times$ size artificial tooth crowns onto silicone 
a

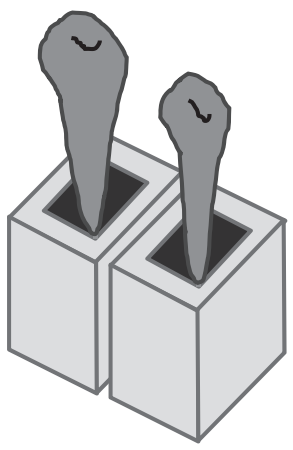

C

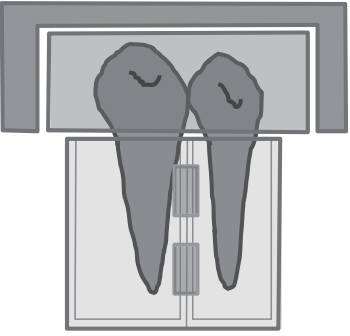

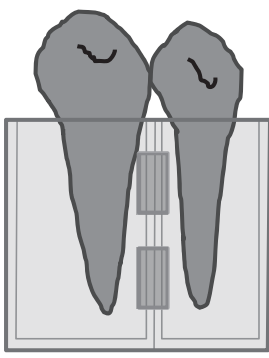

b

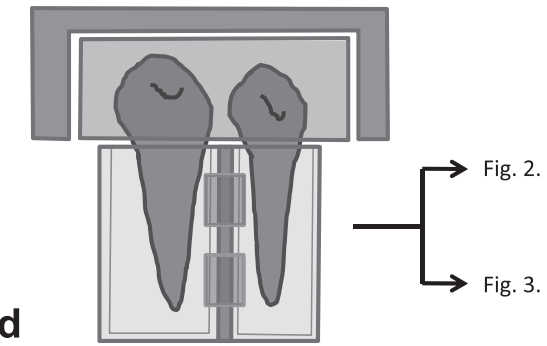

Fig. 1. Manufacture of physical tooth model. a) Teeth were fixed into standardized positioning trays using resin. b) The trays were fixed together using screws, which were then used to adjust the distance between the two tooth crowns. c) A silicone impression was taken with the teeth set at a standardized separation of $0.0 \mathrm{~mm}$. d) A second silicone impression was taken with the teeth set at a standardized separation of $1.0 \mathrm{~mm}$.

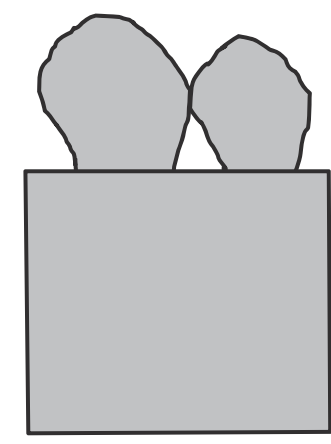

a

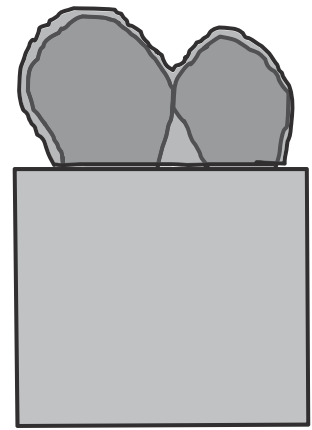

b

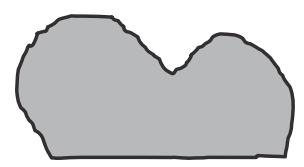

C

Fig. 2. a) A plaster model was produced with teeth set at $0.0 \mathrm{~mm}$ separation. b) Aligner was produced by vacuum-sealing a plastic sheet over the plaster model. c) The aligner created with a coronal proximal surface interval of $0.0 \mathrm{~mm}$.

crown castings with a coronal proximal surface interval of $1.0 \mathrm{~mm}$.

(7) The exposed tooth roots were embedded into a dental silicone impression putty (FUSION II putty type, GC CORPORATION, Tokyo, Japan) (Fig. 3).

(8) The crown silicone impression material was subsequently removed. 


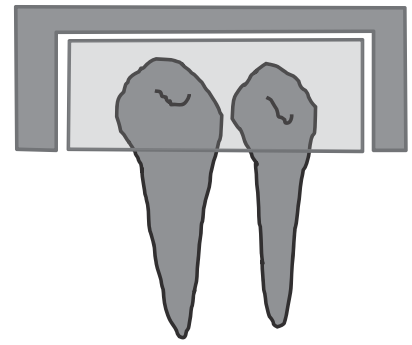

a

Fig. 3. Plastic teeth were set into the silicone impression taken previously with teeth separated by $1.0 \mathrm{~mm}$ (Fig. 1d), and their roots were fixed in silicone dental impression material.

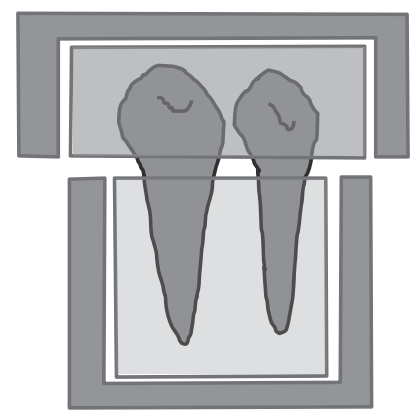

b

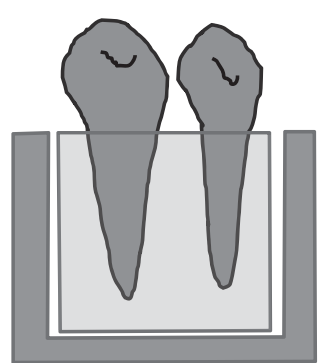

c

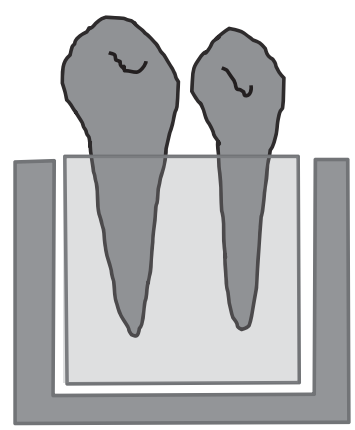

a

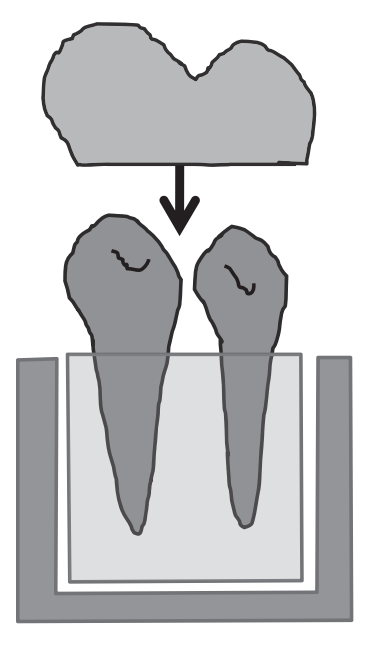

b

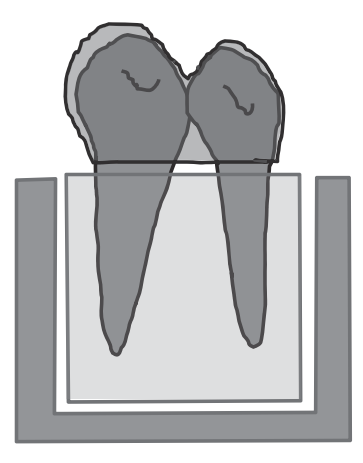

C

Fig. 4. The aligner was vacuum-formed around the model of the two teeth fixed in plaster with a separation distance of $0.0 \mathrm{~mm}$ (Fig. 2a).

(9) The aligner created with coronal proximal surface intervals of $0.0 \mathrm{~mm}$ was then fitted to the artificial tooth model crowns with coronal proximal surface intervals of $1.0 \mathrm{~mm}$ (Fig. 4). When doing this, highly flowable silicone impression material (FUSION II flow type, GC CORPORATION, Tokyo, Japan) was put between the aligner and tooth crowns to visualize where the force was being applied on the crowns (Fig. 5).

(10) Cephalometric photographs were taken of the artificial tooth model with the aligner fitted and imaging was conducted using industrial CT equipment (ScanXmate-L080HT, Comscantecno Corporation, Kanagawa, Japan). We 3D-rendered the tooth crown, root, and alveolar bone shape while the aligner was fitted (Fig. 6). The scanning conditions were $80 \mathrm{kV}$, $20 \mu \mathrm{A}$ (crown area), $90 \mu \mathrm{A}$ (root area), 600 slices / scan, and 48- $\mu \mathrm{m}$ slices.

An FE model was subsequently created from CT DICOM data based on the artificial tooth model. Analysis was performed using mechanical analysis software (Marc, MSC Software Corporation, Sunnyvale, CA, USA) to extract a virtual aligner from shell elements of the FE 

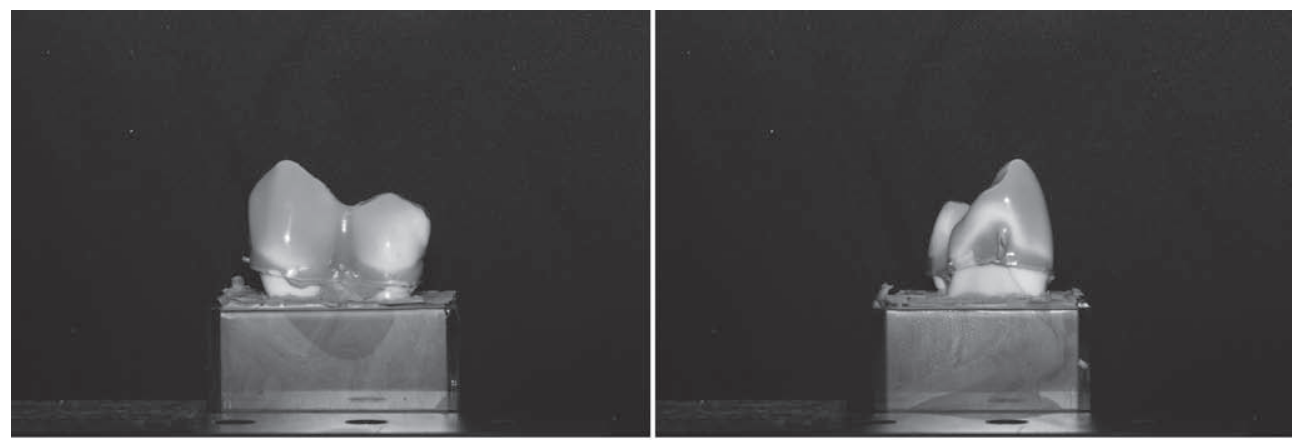

a

b
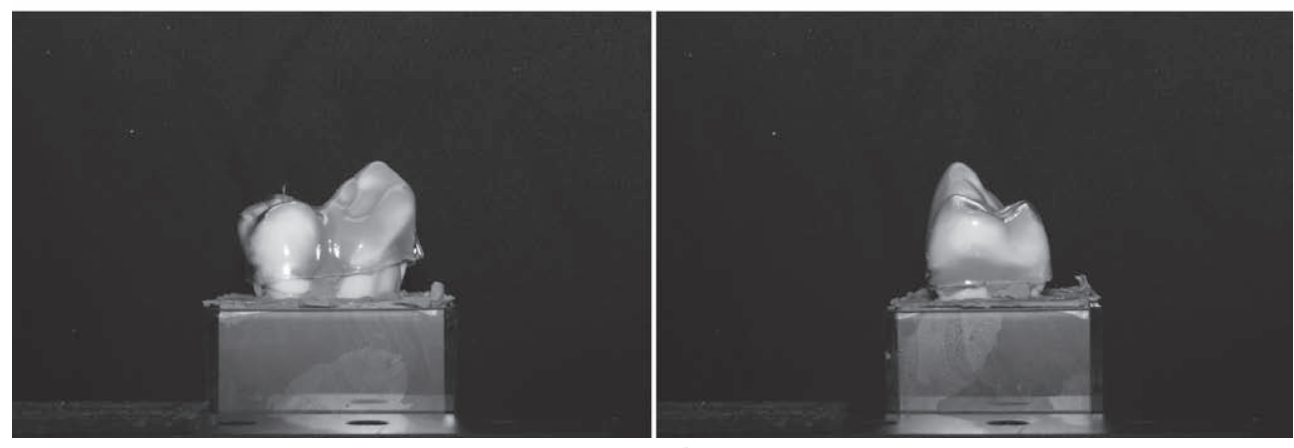

d

Fig. 5. Photographs show the physical model following an impression with flowable silicone impression material between the plastic teeth and the aligner. Areas of light-pink coloration indicate the coronal areas of force application (i.e., the areas where the aligner is tightly fitting against the tooth). Images were taken from different aspects of the model (clockwise from top-left) : a) buccal, b) mesial, c) lingual, d) distal.

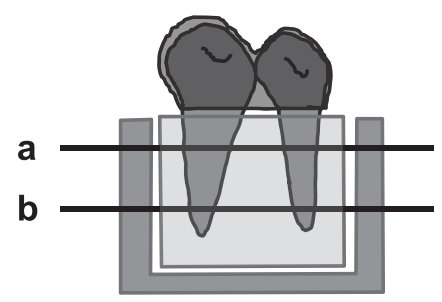

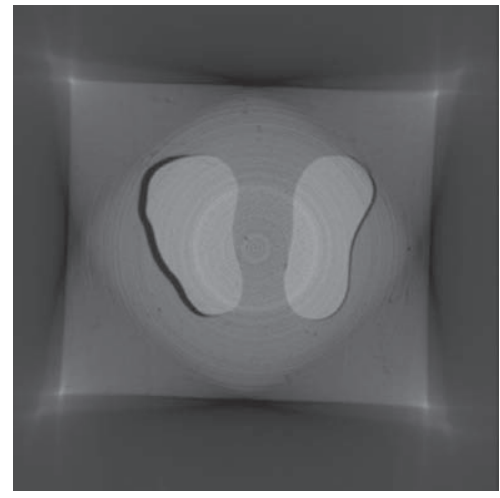

a

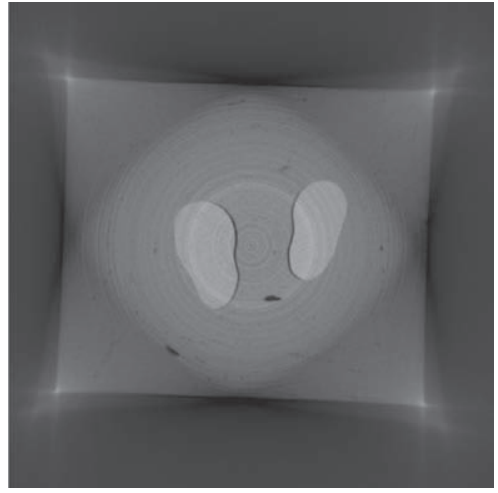

b

Fig. 6. Images show CT reconstructions of the physical tooth model (plastic teeth fixed in silicone impression material). The left side is the canine root and right side, the proximal root. Images represent slices of $48 \mu \mathrm{m}$ thickness. a) Slice from near the cervical end of the roots. b) Slice from near the apical end of the roots. Note the space on the non-proximal side cervically (a), and on the proximal side apically (b). 


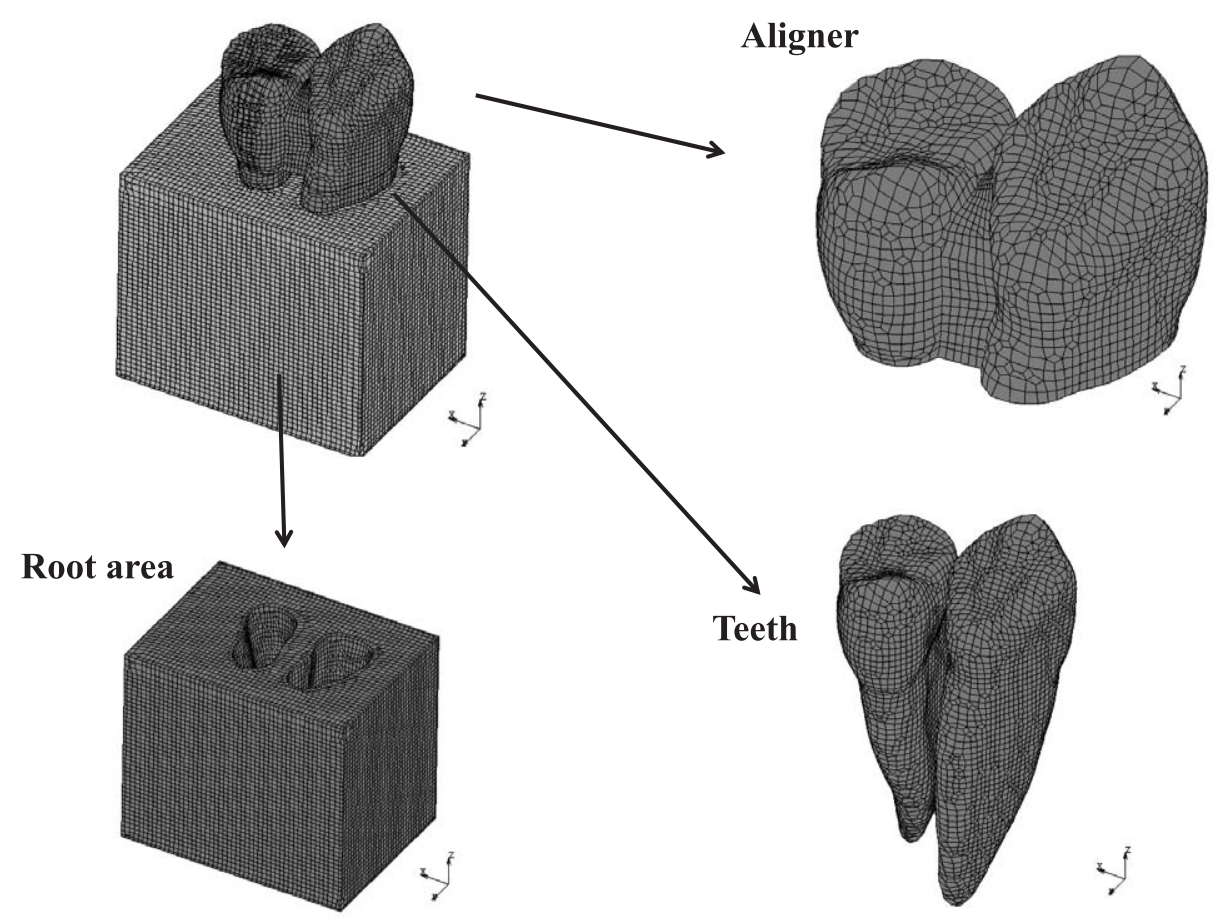

Fig. 7. The finite element analysis was performed by subdividing the model into its component parts: the aligner, the teeth, and the root area.

model crown portions and produce a membrane-like FE model (Fig. 7). Similar to the artificial tooth model, this was fitted to the upper crowns and considered to represent the application of orthodontic force. Constraint conditions were set in the virtual alveolar bone surface and base.

Young's modulus was $21.7 \mathrm{GPa}$ for the teeth, $30 \mathrm{MPa}$ for the putty areas, and $400 \mathrm{MPa}$ for the aligner. Poisson's ratio was set at 0.3 for all materials. There were 21,004 tooth elements, 86,051 putty elements, and 2,846 aligner elements. The teeth, virtual aligner, and putty parts were all in contact and had no common joints.

Next, the virtual aligner was contracted back to its original shape and analyzed to estimate the force points of action from contact points between the virtual aligner and the teeth. We also considered tooth movement from the stress placed on the tooth roots.

\section{Results}

We verified that the force points of action were identical in the artificial tooth model and FE analysis. In the artificial tooth model, pale-colored areas of crown silicone were considered to represent areas subjected to load (Fig. 5). In particular, we observed pale-colored silicone in the maxillary right canine buccal cervical ridge, mesial proximal surface, maxillary right second premolar buccal distal portion, and distal proximal surface areas. We also observed that the aligner and crowns were in contact in approximately the same areas as in the FE analyses (Fig. $8)$.

The CT images of the artificial tooth model also showed gaps near the tooth cervix in the 


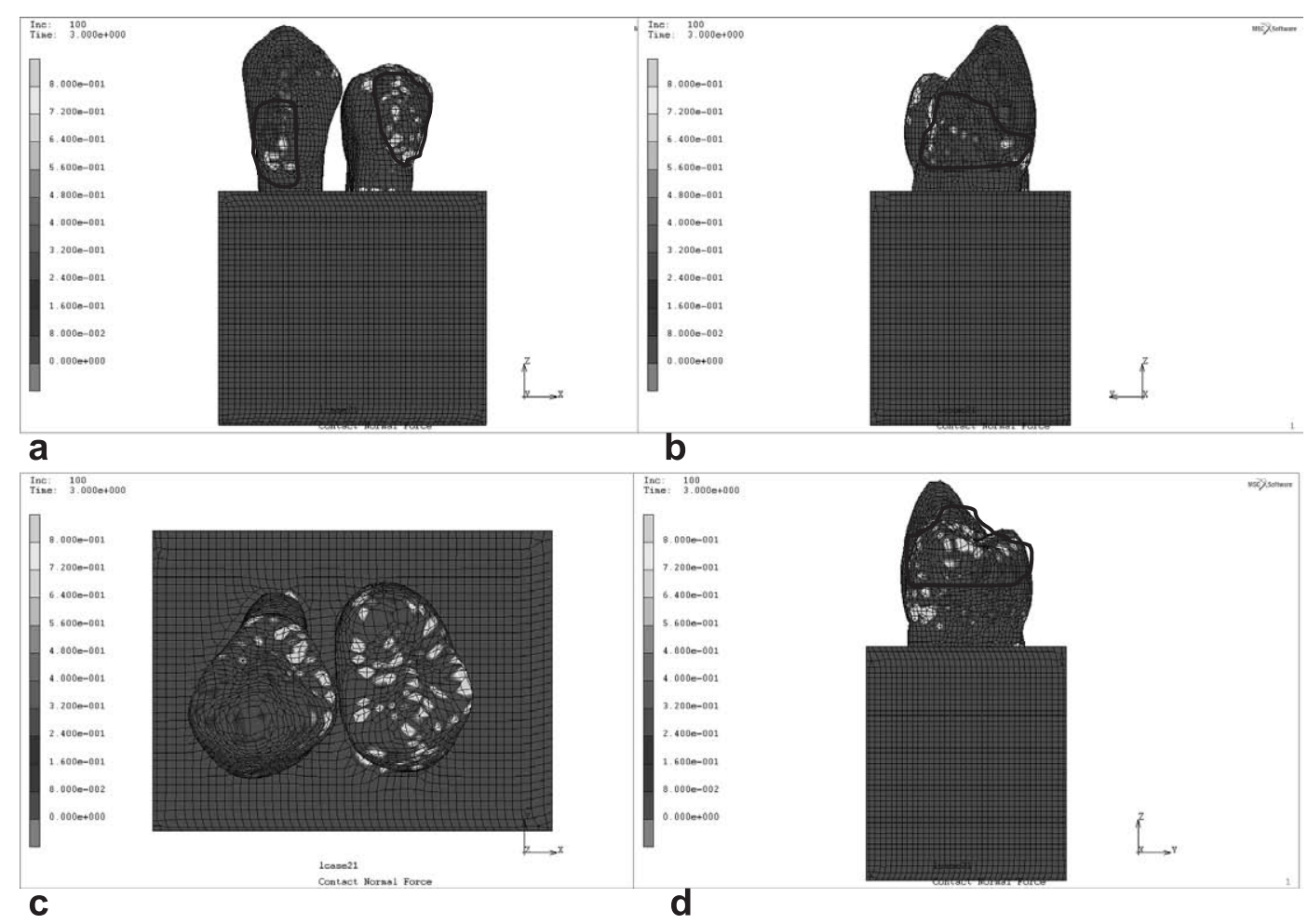

Fig. 8. Results of the finite element analysis. Figures show the distribution of contact points between the tooth surface and the aligner. Close contact points are represented by warm colors (reds, yellows), while looser contacts are shown by cold colors (blues, greens). Images are taken from different aspects of the model (clockwise from top-left) : a) buccal, b) mesial, c) occlusal, d) distal.

maxillary right canine mesial surface and maxillary right second premolar distal surface, as well as near the tooth root in the maxillary right canine distal surface and maxillary right second premolar mesial surface (Fig. 6). Accordingly, we observed a $0.0 \mathrm{~mm}$ crown distance after covering the two teeth that were separated by a $1.0 \mathrm{~mm}$ interval using the aligner, and teeth were moved diagonally towards the gaps.

In addition, we observed a similar tendency in the silicon model and FE analysis, with teeth moving near the tooth cervix in the maxillary right canine mesial surface and maxillary right second premolar distal surface, as well as near the tooth root in the maxillary right canine distal surface and maxillary right second premolar mesial surface (Fig. 9). This finding suggested that tooth root stress is predictable.

\section{Discussion}

Comparison of movement in an artificial tooth model fitted with an aligner and that suggested by $\mathrm{FE}$ analysis in the present study indicated identical results, which demonstrates the validity of using the FE model to mechanically clarify the effects of an aligner on the tooth crown and root. In particular, by creating a contact state and performing mechanical analysis with the teeth and the virtual aligner considered as separate items, we determined exactly where the aligner 


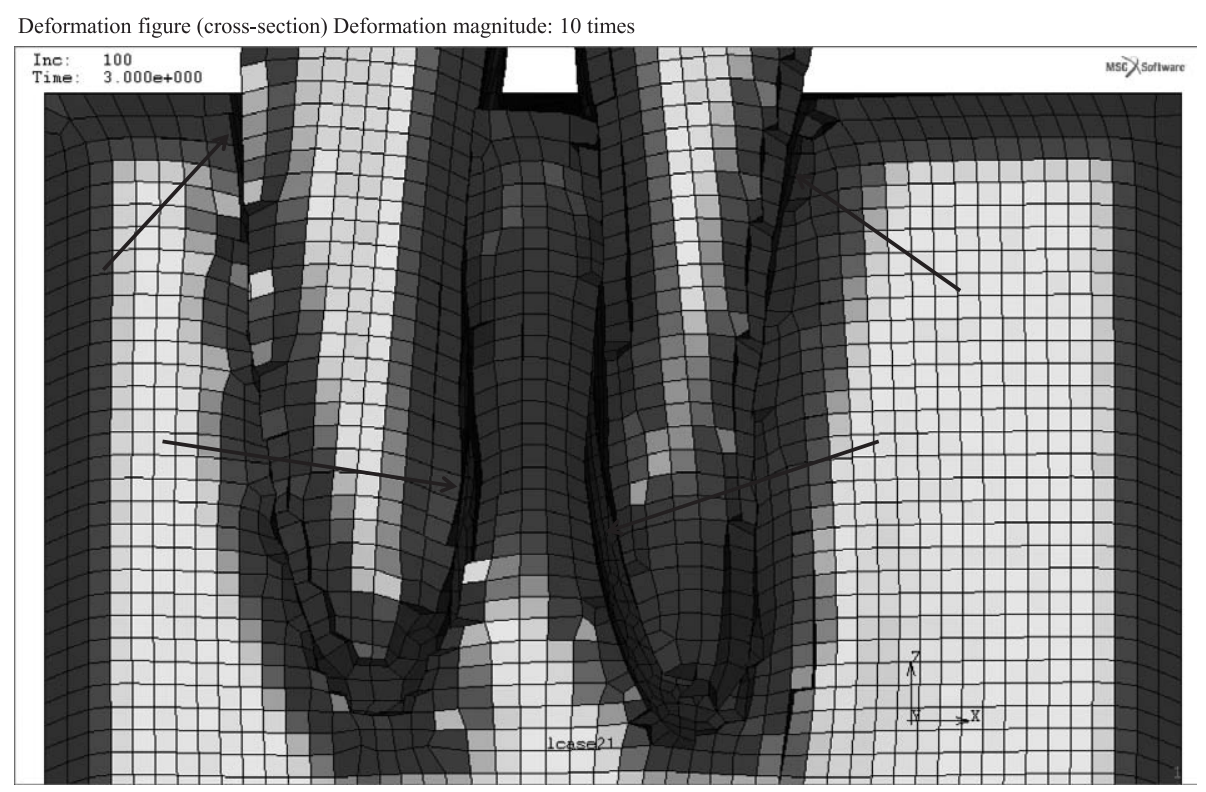

Fig. 9. Enlargement of the tooth root area. Note the space on the non-proximal side cervically, and on the proximal side near the root apex (arrows) as the aligner brings the crowns closer together, consistent with the CT data shown.

was contacting the tooth crowns.

Creating a complex virtual aligner shape with the FE model also allowed us to analyze a state accurately representing the actual model shape. Our results also suggested that in the future, it may be possible to calculate the force applied on teeth and stress placed on tooth roots by aligners. In addition, we could analyze differences in loading due to tooth crown shape and differences in movement directions. In the future, mechanical analysis will be vital for more accurate simulations in clinical settings.

There are still limitations to computer processing capabilities in mechanical simulations of patient dentition. Furthermore, alveolar bone dynamic processes must be accurately substituted into an FE analysis from CT results, requiring the popularization of a technique for correcting cone-beam CT values ${ }^{12)}$. However, if continuous output of mechanical analysis results becomes possible, tooth movement could also be viewed in video form ${ }^{13)}$, and simple image simulations may evolve into simulations reflecting actual mechanical behavior.

\section{Conflict of interest}

The authors have declared no conflict of interest.

\section{References}

1) Proffit WR. Malocclusion and Dentofacial Deformity in Contemporary Society: The Changing Goals of Orthodontic Treatment. In Proffit WR, Fields HW Jr, Sarver DM, et al, eds. Contemporary Orthodontics. 5th ed. St. Louis: Mosby Elsevier; 2013. pp 2.

2) Maia LG, de Moraes Maia ML, da Costa Monini A, et al. Photoelastic analysis of forces generated by 
T-loop springs made with stainless steel or titanium-molybdenum alloy. Am J Orthod Dentofacial Orthop. 2011;140:e123-e128.

3) Kurol J, Franke P, Lundgren D, et al. Force magnitude applied by orthodontists. An inter- and intra-individual study. Eur J Orthod. 1996;18:69-75.

4) Canales C, Larson M, Grauer D, et al. A novel biomechanical model assessing continuous orthodontic archwire activation. Am J Orthod Dentofacial Orthop. 2013;143:281-290.

5) Cattaneo PM, Dalstra M, Melsen B. The finite element method: a tool to study orthodontic tooth movement. $J$ Dent Res. 2005;84:428-433.

6) Mo SS, Kim SH, Sung SJ, et al. Factors controlling anterior torque during C-implant-dependent en-masse retraction without posterior appliances. Am J Orthod Dentofacial Orthop. 2011;140:72-80.

7) Kojima Y, Fukui H. Numeric simulations of en-masse space closure with sliding mechanics. Am J Orthod Dentofacial Orthop. 2010;138:702, e1-e6.

8) Sung SJ, Jang GW, Chun YS, et al. Effective en-masse retraction design with orthodontic mini-implant anchorage: a finite element analysis. Am J Orthod Dentofacial Orthop. 2010;137:648-657.

9) Kim T, Suh J, Kim N, et al. Optimum conditions for parallel translation of maxillary anterior teeth under retraction force determined with the finite element method. Am J Orthod Dentofacial Orthop. 2010;137:639-647.

10) Boyd RL, Miller RJ, Vlaskalic V. The invisalign system in adult orthodontics: mild crowding and space closure cases. J Clin Orthod. 2000;34:203-212.

11) Nagara Y, Koseki M, Shimada S, et al. A biomechanical study of orthodontic forces generated by thermoplastic orthodontic appliance using CAD / CAM system. J Oromax Biomech. 2008;14:14-19. (in Japanese).

12) Baba R, Ueda $\mathrm{K}$, Takahashi $\mathrm{M}$, et al. Scattered X-ray correction method for cone-beam CT. MED IMAG TECH. 2009;27:177-184. (in Japanese).

13) Fujita Y, Maki K. A three-dimensional finite element stress analysis of thermoplastic orthodontic appliances treatment. Annu Meet Jpn Orthod Soc. 2013;72:174. (in Japanese).

[Received February 4, 2014 : Accepted April 21, 2014] 